\title{
Über Raupen, Blumenwiesen und Kreidekreise
}

\author{
Festansprache zur ersten Bachelor-Graduierungsfeier der Elementarpädagog*innen \\ Mi., 29. Sept. 2021, PH NÖ
}

Erwin Rauscher ${ }^{1}$

\section{Zusammenfassung}

Der Beitrag ist die schriftliche Fassung der Festansprache des Rektors anlässlich der ersten BA-Graduierungsfeier im Rahmen des neuen Elementarpädagogikstudiums an der PH NÖ. Es gilt das gesprochene Wort!

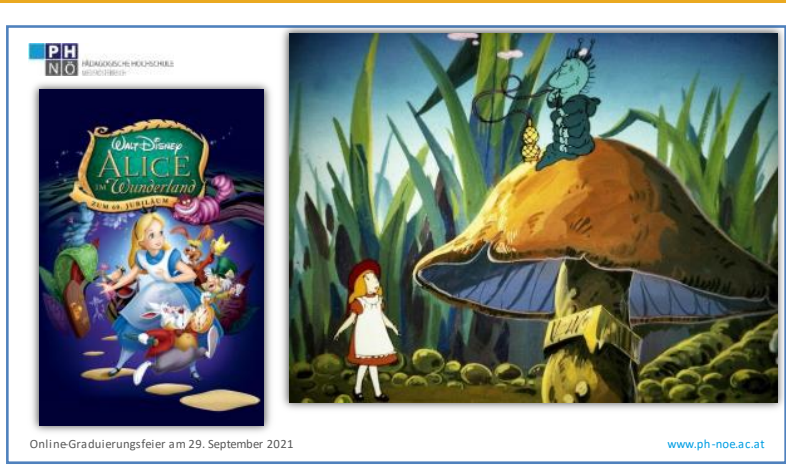

„Wer bist denn du?" sagte die Raupe. „Ich weiß es selbst kaum", sagte Alice, „ich muss seit dem Aufstehen heute früh wohl mehrere Male vertauscht worden sein. "Wie meinst du das?" fragte die Raupe streng. „Erklär dich!" „Ich fürchte, ich kann mich nicht erklären", sagte Alice, "denn ich bin gar nicht ich, sehen Sie." "Ich sehe es nicht", sagte die Raupe.

Mit diesem seltsamen Wort aus Alice in Wonderland begrüße Sie alle herzlich in dieser Feierstunde. Und beglückwünsche Sie, geschätzte Bachelors of Education, gemeinsam mit Ihren Partnern, Verwandten, Freunden, um Ihnen allen zu gratulieren, in ferner Nähe.

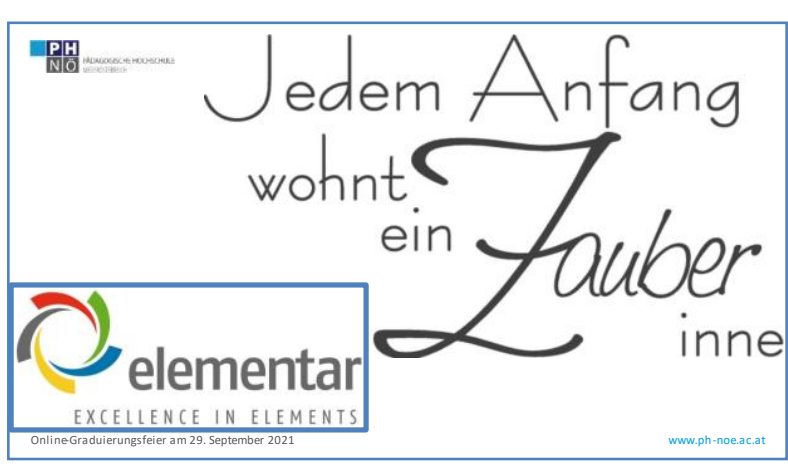

So viel Anfang war noch nie - Hölderlin. Sie sind unsere erste Gruppe in der Elementarpädagogik mit akademischem Abschluss. Elementarpädagogik - ich musste erst nachdenken, was hinter diesem inzwischen so geläufig gewordenen Wort eigentlich steckt.

Elementar - das heißt doch nicht nur einfach und simpel und anfängerhaft, sondern auch grundlegend: Die Naturgesetze etwa sind elementar für unser Weltbild. Ein Elementarereignis nennen wir ein Naturwunder, ebenso wie einen Supergau mit seiner Elementargewalt. In der Mathematik heißt die Menge aller Elementarereignisse Ergebnismenge eines Zufallsexperiments. Wenn wir in der Physik von den kleinsten Teilchen sprechen, die es überhaupt gibt, und von den Grundkräften, die diese kleinsten Teilchen zusammenhalten, denn betreiben wir Elementarteilchenphysik. In grauer Vorzeit bis hinein ins Mittelalter gab es die Vier-Elemente-Lehre mit Feuer, Wasser, Luft und Erde. Und bis heute in der Chemie nennen wir jene Stoffe, die mit chemischen Methoden nicht mehr in andere Stoffe zerlegt werden können, die Grundstoffe aller chemischen Reaktionen - Elemente.

Und Pädagogik, das haben Sie ja längst gelernt, setzt sich aus pais, das Kind, und agein, führen und leiten, zusammen.

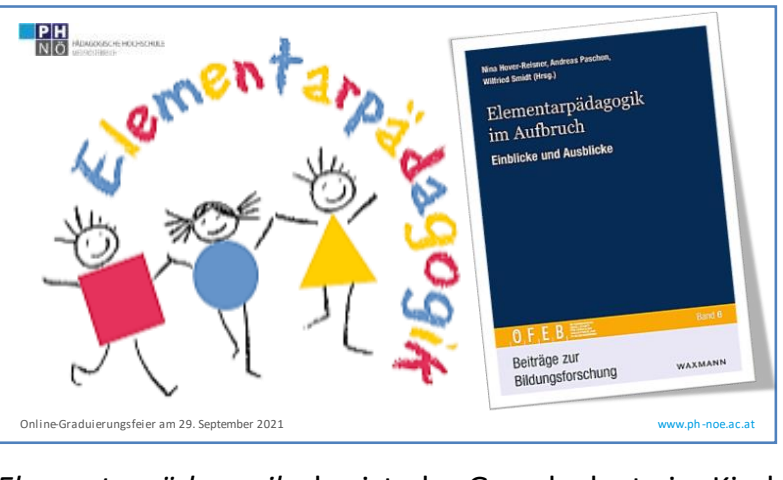

Elementarpädagogik also ist, das Grundgelegte im Kind zu entbergen, anzustiften, auch anzuleiten, zu begleiten, zu entwickeln, auch zu entfremden. Mir ist heute früh für Sie aber noch ein zweites Wort in den Sinn ge-

\footnotetext{
${ }^{1}$ Univ.-Prof. MMag. DDr. Erwin Rauscher ist Rektor der Pädagogischen Hochschule Niederösterreich, Mühlgasse 67, 2500 Baden.E-Mail: erwin.rauscher@ph-noe.ac.at
} 
kommen, das so international ist, dass es selbst im Englischen genau gleich heißt, nämlich: Kindergarten. Und ich habe über dieses zweite Kompositum nachzudenken begonnen: Was ist denn eigentlich das Wesen des Kindseins? Welcher Art ist dieses Sein des Kindes? Welcher Sinn liegt darin?

Kindheit darf doch kein Stadium sein, das möglichst schnell aufhört, dumm zu sein, das uns Erwachsene stört, das sich erst durch Bildung und Wachstum zur vollen Entfaltung des Menschseins löst, das Frauenangelegenheit ist und deshalb talibanischer Männer unwürdig.

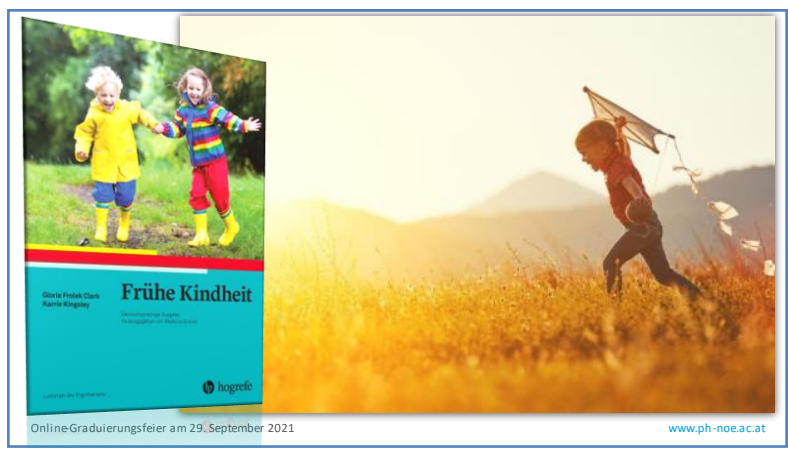

Meine erste Erkenntnis also: Kindheit ist kein Stadium, das überwunden werden muss. Kindheit und Anfang sind nicht Leere, sind nicht Nichts, sind nicht ein Etwas, das sein Sein außer sich hat. Kindheit ist vielmehr das an sich, was der Erwachsene für sich ist. Das aber heißt, ein Erwachsener kann nur von seinem Kindsein her - und mit diesem - Aussagen treffen. Kindheit ist Sein als Werden! Kindsein ist ein Ja zur Gegenwart, nicht aber ein Reden von einer Zukunft, die nicht erwartet werden kann. Sich auf ein Kind einzulassen heißt anfangen, ein Mensch zu sein.

Und weil ich an der PH ständig über das Unterrichten in der Schule rede, habe ich mich gefragt, ob man das mit dem Spielen im Kindergarten vergleichen kann. Ist es nur ein Vorspiel für den Ernst des Lebens? Jedenfalls ist es kein Gegensatz zur Arbeit - wie vielleicht viel später. „Wenn sie nicht hören, reden, fühlen noch sehen, was tun sie dann? Sie spielen“, sagt der Fabeldichter Magnus Gottfried Lichtwer.

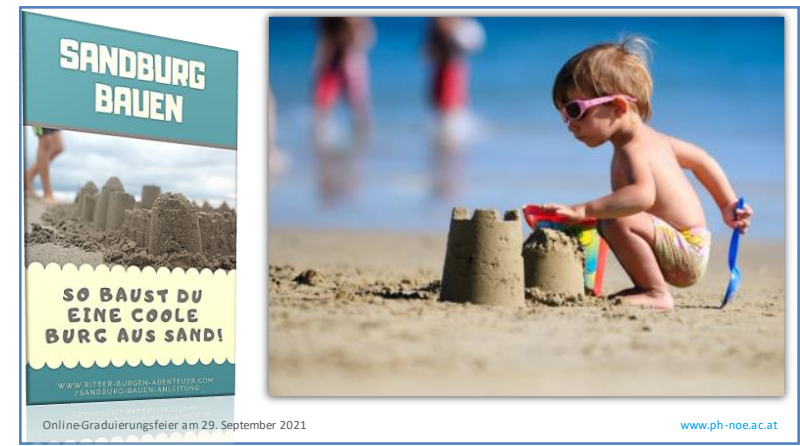

Wenn meine Enkelkinder eine Sandburg gebaut haben und ich ihnen zu helfen versucht habe, damit alles wunderschön werde, zertreten sie sie plötzlich, während ich noch überlege, wie man sie für den nächsten Tag aufbewahren könnte, und beginnen eine neue zu bauen.

Ein Kind kann lassen, es kann loslassen. Seine Sandburg ist kein Haben, es hält nicht fest. Es kann lassen, weil es sich bewahrt weiß. Ein spielendes Kind denkt nicht vor, es schaut nicht nach. Es kümmert sich recht wenig um die Urteile der Entwicklungspsychologen, die sein Spiel deuten, als Abreagieren, Erholung, Einübung, Nachahmung oder gar Flucht in die Illusion. Es versucht stundenlang, ein selbst gestecktes Ziel zu erreichen, weint und bitzelt, aber es lässt sich nicht helfen, will sein Angewiesensein auf helfende Hände überwinden, sein eigenes Ich sein, will es selbst sein. Ist der Kindergarten nicht der Gelegenheits-Ort, um selbst jemand werden zu können?

Erlauben Sie mir dazu ein Beispiel, das Sie sicher alle kennen:

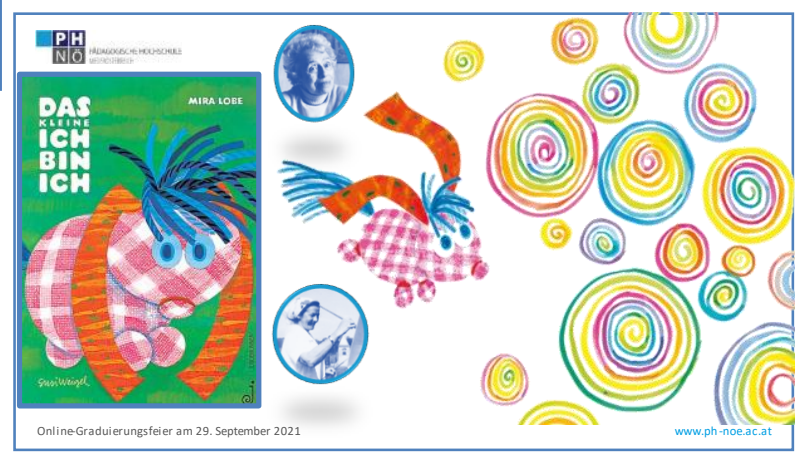

Das Buch mit Texten von Mira Lobe und Zeichnungen von Susi Weigel - es wird jetzt bald 50 Jahre alt. Es wurde mit dem Österreichischen Staatspreis für Kinder- und Jugendliteratur ausgezeichnet, ist in zahlreiche Sprachen übersetzt und vielfach neu aufgelegt worden. Es dient als Vorlage für Theaterstücke, MusikCDs, Computerprogramme. Eben erst erschienen ist die erste dreisprachige Ausgabe - auf Deutsch, Arabisch und Farsi - sie soll das Deutschlernen und somit auch die Integration erleichtern. Sein Text klingt wie ein balladenähnliches Langgedicht und beginnt so:

Auf der bunten Blumenwiese geht ein kleines Tier spazieren, wandert zwischen grünen Halmen, wandert unter großen Palmen, freut sich, dass die Vögel singen, freut sich an den Schmetterlingen, freut sich, dass sich`s freuen kann. Aber dann... Aber dann stört ein Laubfrosch seine Ruh und fragt das Tier: "Wer bist denn du?" Da steht es und stutzt und guckt ganz verdutzt dem Frosch ins Gesicht: „Das weiß ich nicht." 
Der Laubfrosch quakt und fragt: „Nanu?

Ein namenloses Tier bist du?

Wer nicht weiß, wie er heißt, der ist dumm!"Bumm.

Verzweifelt fragt es nun andere Tiere - Pferde, Fische, weiße Vögel, Nilpferde, Papageien, Hunde - ob jemand weiß, wer es sei. Aber keiner weiß es.

Durch die Stadt und durch die Straßen

geht das bunte Tier spazieren;

geht und denkt so vor sich hin:

"Stimmt es, dass ich gar nichts bin?

Alle sagen, ich bin keiner, nur ein kleiner irgendeiner...

Ob es mich etwa gar nicht gibt?"

Und das kleine bunte Tier,

das sich nicht mehr helfen kann,

fängt beinah zu weinen an.

Aber dann ...

Aber dann bleibt das Tier mit einem Ruck,

mitten im Spazierengehen,

mitten auf der Straße stehen

und es sagt ganz laut zu sich:

"Sicherlich gibt es mich: ICH BIN ICH!"

Das kleine Ich-bin-ich freut sich - und es gibt seine Erkenntnis sogleich an alle anderen Lebewesen weiter. Wir sehen: Unser buntes, kugeliges Tier ist auf der Suche nach seiner Identität. Seine Geschichte ist eine Hommage an die Entdeckungslust des Kindes, vor allem aber ein Loblied auf die Individualität jedes Einzelnen. Es dient für alle Kinder als Identifikationsfigur - es ist ein buntes Tier, das fliegen und auf einer Wolke schlafen kann, und doch ist es auch ein Sonderling und Außenseiter in der Welt der Tiere.

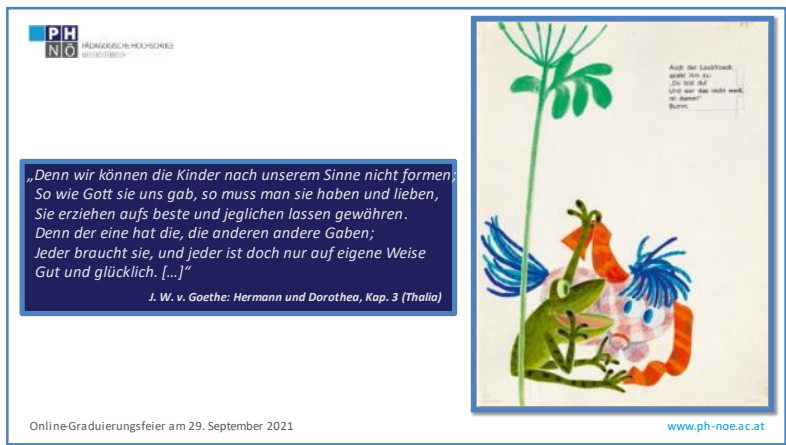

Der Frosch will es anfänglich nicht einordnen und schließt sich damit selbst aus: Indem er exklusiv festlegt, dass derjenige dumm sei, der nicht weiß, wohin er gehört und wer er ist. Unser kugeliges Tier aber überwindet seine Existenzkrise, indem es die ontologische Frage, ob es denn niemand sei, mit seiner Selbsterkenntnis beantwortet, auf die genau ihm zugehörigen Eigenschaften und Seinsweisen zurückgreift und so die Lösung findet. Das stößt bei den anderen Tieren auf Akzeptanz - auch durch den Frosch wird es rehabilitiert. Nicht länger namenlos, voller Selbstbewusstsein, akzeptiert und integriert, nicht länger ausgeschlossen.
Es bekommt es zur Antwort: „Du bist du!“ Mit sich selbst zufrieden, stellt sich auch die Harmonie mit der Natur wieder her: Es „freut sich an der schönen Welt, die ihm wieder gut gefällt."

Und parallel zu dem Leitwort inklusiver Pädagogik von Goethe, aus Hermann und Dorothea hier auf der Folie, erlauben Sie mir einen pädagogischen Nebensatz zum Stichwort „Lerngesundheit“: Wenn sich ein Kind als sozial unerwünscht erlebt, als last preferred co-worker, als eines, das aufhält, anstatt gebraucht zu werden, dann wird dieses Kind aus der verlorenen Sicherheit, für andere Menschen wichtig zu sein oder nur so viel zu gelten, wie es kann, nicht so viel, wie es ist, entweder selbst kränkeln oder andere kränken, wenn nicht sogar beides. Anders gesagt: Kinder, die gehorchen müssen, aber nicht geachtet werden: sie kränkeln selbst, und sie kränken andere!

Achtung und Wertschätzung unabhängig von Leistung! Würde und Güte unabhängig von Disziplin. Das ist der Hauch von Inklusion. Wenn Sie etwas für diese Selbstwirksamkeitserwartung tun, reduzieren Sie die Gewalt der Ohnmacht gegen subjektiv erlittene Willkür im Kindergarten.

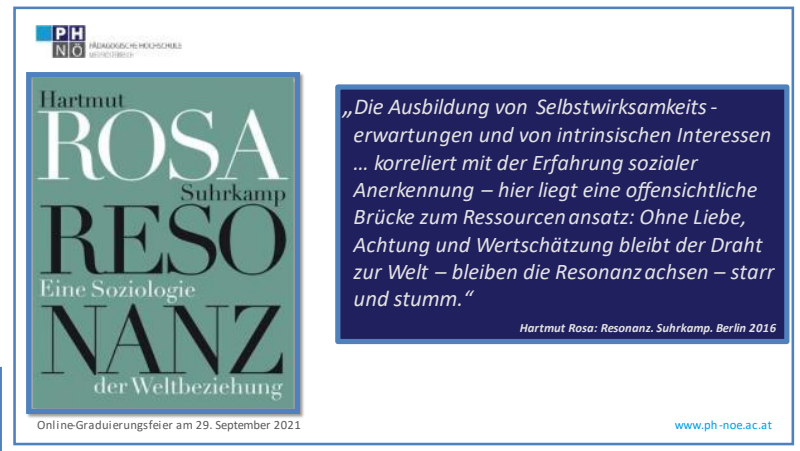

Ungleich präziser aber als ich das kann, hat es der Soziologe Hartmut Rosa von der Friedrich-Schiller-Universität Jena in seinem Buch „Resonanz" formuliert. Es ist 2016 bei Suhrkamp erschienen - mittlerweile bereits in 4. Auflage - Sie können es ja selbst lesen.

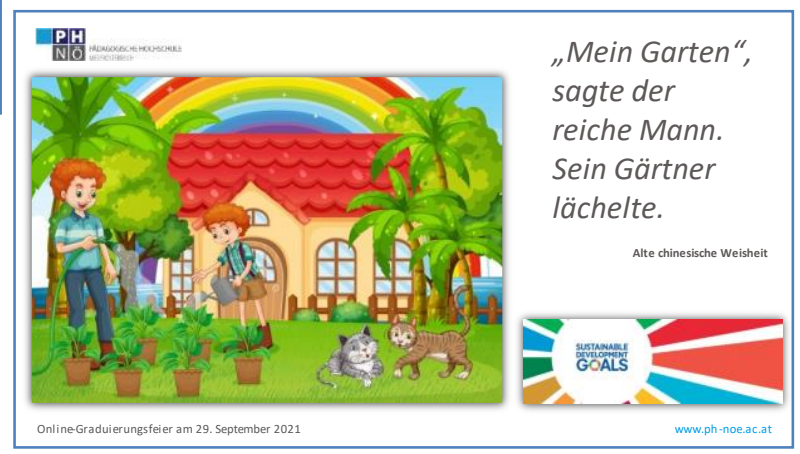

Jetzt habe ich viel über Kindsein und Kindheit gesagt vom Kindergarten fehlt noch der Garten zum Kompositum. Nun könnte man zeithörig sagen: Gärtner*innen sind ausgebildete Fachleute für Produktion, Ernte, Pfle 
ge von Pflanzen, zur Freude und zum Wohl der Menschen. Ihre Fachrichtungen reichen von der Baumschule über Garten- und Landschaftsbau, Gemüse- und Obstbau, Stauden- und Zierpflanzenbau bis hin zur Friedhofsgärtnerei. Daraus folgt für Sie heute ein neuer Auftrag für die Kindergärten, nämlich die Kinder in kindgerechter, in altersgerechter Form zu den SGDs hinzuführen - was das im Kindergarten bedeutet, wage ich nicht einzuschätzen, aber es wäre vielleicht ein spannendes Bachelorarbeitsthema.

Aber ich reduziere es heute auf eine ganz einfache $\mathrm{Pa}$ rabel, die noch dazu einfach stehen lassen und nicht erklären werden - ich habe sie Ihnen einfach hingeschrieben.

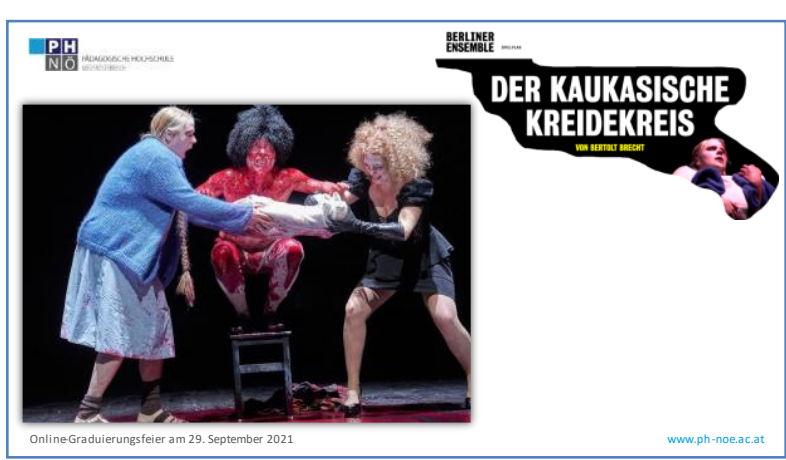

Bevor ich nun zum Schluss komme, lassen Sie mich Ihnen noch ein letztes Beispiel aus der Literatur aufzeigen, das mir für Ihr pädagogisches Tun an den Elementen des Wachsens und Lebens persönlich ganz wichtig ist ... hin zu Bertolt Brechts pädagogischem ParadeLehrstück, dem Kaukasischen Kreidekreis: Sie alle kennen gewiss die Geschichte vom Richter Salomon, der zu entscheiden hat, ob das von ihr weggelegte Kind der leiblichen Mutter, einer reichen Frau, oder der armen Magd gehört, die es aufgezogen hat. Die Geschichte dieser Magd, die das weggelegte Kind ihrer Herrin aufzieht und, als diese es wiederhaben will, vor den Richter tritt, der das Kind in den Kreis stellt und zerteilen will, um beiden gerecht zu werden. Doch die Magd lässt los, sie will das Kind nicht aufgeteilt in seine Eigenschaften, sondern ganz behalten. Und dafür gibt sie es her. Es ist die Geschichte vom salomonischen Urteil noch vor aller Elementarpädagogik. Sie wird von einem Sänger erzählt, der sie mit einem seltsamen Wort beginnt: Schrecklich ist die Verführung zur Güte.

Zuhause, in Ihren Kindergärten, weit weg von Ihrem Studium hier bei uns an der Hochschule mit all seinen Modewörtern pädagogischer Bildung, aber ganz nahe bei den anvertrauten Kindern, könnte jener Sänger unseren Lehrpersonen das unzeitgemäße Wort Brechts zurufen: Es verläuft das Kälbchen sich, wenn der Hirte schläft.

Bei Brecht - zurzeit wird das Stück am Berliner Ensem-

ble ja wieder gespielt, mit der österreichischen Hauptdarstellerin Stefanie Reinsperger als Magd Grusche. Der betrunkene Richter fragt die Grusche, auf den Buben zeigend: Was für ein Kind ist es? So ein verlumpter Straßenbankert oder ein feines, aus einer vermögenden Familie? Und Grusche antwortet: Es ist ein gewöhnliches. Liebe, Zuneigung, Fürsorge und Solidarität sind gewöhnlich. Sie sind Zeichen der Vernunft, sie leben in jedem vernünftigen Menschen. Es sind jene Soft Skills, die Sie in Ihren Kindergärten allemal besser spüren als jede Erziehungswissenschaftlerin, nämlich täglich in ihren Gruppen.

Mitten in der Gerichtsverhandlung bittet die Magd den Richter Azdak: "Wenn ich's [das Kind] nur behalten könnt, bis es alle Wörter kann. Es kann erst ein paar."

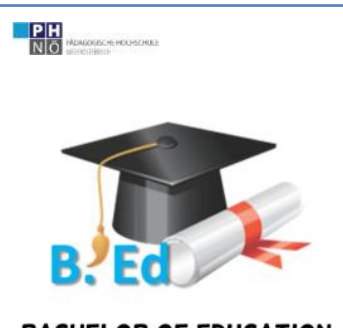

BACHELOR OF EDUCATION

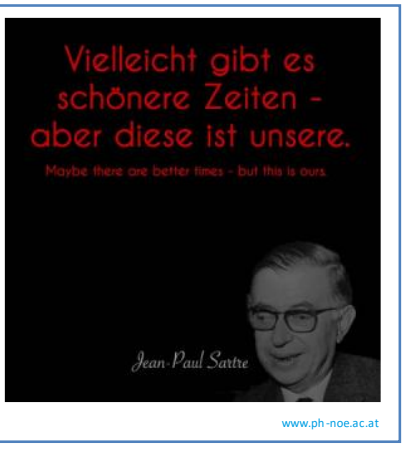

Und mit diesem Satz von Jean Paul Sartre sage ich: Vielleicht gibt es noch schönere Tage - aber dieser ist Ihrer. Ihr Fest-Tag am Ende des Studiums. Wenn auch nur als virtuelles Dekret, bekommen Ihr Zeugnis heute ... als Menschen für neue Freiheit und Verantwortung.

Wenn Sie es dann mit der Post erhalten, werden Sie es anschauen, sich hoffentlich noch einmal freuen ... und dann werden Sie Ihr Zeugnis einmal weglegen, später nicht mehr oft aufnehmen ... und doch werden Sie Zeugnis davon ablegen, ein Leben lang. Sie geben Zeugnis durch Ihr Tun. Nicht wer kennt oder bekennt: Nur wer tut, gibt wirklich! So ist es auch in der Liebe.

Ihr Zeugnis besteht darin, dass Sie Zeugnis ablegen. Und diese Verantwortung für die Erziehung der Kinder zur Freiheit können Sie teilen - mit den Kindern selbst und mit deren Elternhäusern: Von Geburt an ist Kindsein Leben, um das Lieben zu lernen. Kindergartenkind sein ist Lernen, das Leben zu lieben. Mutter und Vater sein ist Lieben, um das Leben zu lehren. Elementarpädagogin sein ist Lehren, die Liebe zu leben.

Aber damit es nicht zu ernst endet, und weil aller Anfang auch schwer ist, könnte es ja sein, dass Sie heute nach dieser Feierstunde beim privaten Feiern voll Freude dem Opapa erzählen Ich hab' jetzt den BEd, dann soll der covid-verängstigte Opa nicht antworten: Aber bitte steck mich nicht an! 
Sie also scheinen am Ende Ihres Studiums angekommen zu sein. Und da gilt: Ende gut, Anfang gut! Wer am Ende ist, der kann von vorne anfangen. Das Ende Ihres Studiums kann neuer Anfang und Aufbruch sein: Und dabei nie wieder Ankommen: Denn Ankommen geht vorüber. Aufbrechen ist ewig. Also brechen Sie auf! Und ich breche jetzt ab - sonst brechen Sie weg!

Für Ihre kleinen Königskinder, Ihre schutzwürdigen Schutzbefohlenen, wünsche ich Ihnen Gottes Segen! 